\title{
Crítica a las políticas de desarrollo regional en Colombia en el marco de la globalización: el caso del departamento de Córdoba
}

\author{
Jorge Eliécer Ortega-Montes*
}

\begin{abstract}
In this work the study is focused on analyzing regional policies in Colombia from the early stage of the period concentrated on substituting imports to the current times of free market and competitive production, having as a case the Department of Cordoba, in the Colombian Caribbean region. Its theoretical underpinnings have been analyzed and the corresponding level of responsibility in terms of the social inequalities resulting from its implementation. This study is expected to contribute to other case studies or studies in the field and to the debate about the making of an alternative model for development with criteria for equity, social justice, and democratic participation.
\end{abstract}

Keywords: regional developmental policies, free market, globalization and competitive production, ownership policies and reforms, violence and dislocation, inequality and development.

\section{Resumen}

En este trabajo se examina el comportamiento de la política regional en Colombia desde la época del modelo sustitutivo de importaciones hasta la actual era de la apertura y la competitividad, a partir del caso del departamento de Córdoba, región Caribe, analizando los fundamentos teóricos que la soportan y el grado de responsabilidad que ésta tiene en las desigualdades sociales generadas por su implementación. Se espera que ello sirva de base para otros casos o estudios similares y contribuya al debate sobre la construcción de un modelo alternativo de desarrollo con criterios de equidad, justicia social y democracia participativa.

Palabras clave: políticas de desarrollo regional, apertura, globalización y competitividad, reforma agraria y tenencia de la tierra, desplazamiento forzoso, desigualdad y desarrollo.

\footnotetext{
*Universidad de Córdoba, Montería, Colombia. Correo-e: jorgeortega@escarsa.net.co.
} 


\section{Introducción}

Actualmente, cuando en Colombia y América Latina se debate sobre los problemas del desempleo, la pobreza, la calidad de vida y la falta de oportunidades que afectan a un gran número de la población, y cuando se presume que muchos de tales problemas tienen su explicación en las políticas macroeconómicas y regionales y en fenómenos internacionales, los estudios locales regionales cobran importancia en tanto que esta escala territorial se constituye en el principal espacio donde convergen las fuerzas de la economía y se construyen las bases de un nuevo ordenamiento internacional.

En este marco de problemas y controversia cabe preguntarse sobre la pertinencia, relación y grado de responsabilidad que las políticas económicas regionales tienen sobre la situación del departamento de Córdoba, región Caribe de Colombia, principalmente en el periodo 1995-2004, que permita proponer alternativas.

Para este efecto se abordan los fundamentos teóricos y los principales instrumentos de la política económica regional en Colombia, enfatizando en sus contradicciones e incoherencias teórico-prácticas y estratégicas.

Es necesario aclarar que este documento no es un estudio de convergencia o divergencia económica regional ni de políticas o enfoques regionales, tampoco de historia económica regional ni de factores determinantes del crecimiento económico local. Se trata simplemente de una aproximación empírica que busca identificar evidencias sobre el comportamiento de la política económica regional en Colombia, escudriñando sus tendencias, implicaciones y oportunidades.

\section{Bases conceptuales y supuestos}

Todo análisis o reflexión sobre los problemas del desarrollo encierran cierto tipo de conceptos teóricos y metodológicos que lo sustentan, los cuales pueden ser explícitos o quedar implícitos en la respectiva argumentación que se derive.

En el caso que nos ocupa, los conceptos y supuestos básicos que orientan el análisis son los siguientes.

La desigualdad del desarrollo entre distintos países o naciones se da también entre los diferentes grupos sociales u organismos de cada nación. 
Los esquemas teórico-metodológicos y la clasificación de las tendencias históricas del desarrollo y de su medición, no son más que abstracciones que sirven de guía para la investigación, comprensión y explicación de la realidad, razón por la cual no todos los elementos u organismos que participan en ellas han pasado, o necesariamente tienen que pasar, por las distintas etapas y condiciones que las caracterizan.

El problema regional objeto de esta reflexión se concibe en términos de diferenciación de oportunidades y acceso de la población al trabajo, calidad de vida, ingresos y toma de decisiones en los asuntos que les competen, en el entendido de ver el territorio como condición y reflejo de las relaciones sociales y del desarrollo social (Coraggio, 1977, 1979, 2001; Triveli, 1995).

\section{Evolución y trascendencia del concepto de desarrollo}

Sin pretender agotar la variada gama de definiciones al respecto, bien se sabe que el concepto de desarrollo proviene desde la Antigüedad, cuando ya se usaba para significar un proceso de realización de acuerdo con ciertas normas preestablecidas. Cornelius Castoriadis (1991: 96) sostiene que un organismo se desarrolla cuando progresa hacia su madurez biológica y que una idea se desarrolla cuando se explica todo aquello que se cree que esa idea contiene implícitamente. Para este autor, el desarrollo implica la definición de una madurez y luego el de una norma natural: el desarrollo, dice, no es más que otro nombre de la fisis aristotélica, que la naturaleza contiene sus propias normas como fines hacia los cuales los seres se desarrollan y que alcanzan efectivamente.

Desde el punto de vista geopolítico, el concepto de desarrollo trasciende las fronteras nacionales desde mediados del siglo XVIII, cuando ya se empieza a celebrar y propagar por el mundo el progreso alcanzado por los países industrializados de Europa Occidental; época en la cual el crecimiento económico es sinónimo de desarrollo, y cuya orientación se deja a la suerte del libre mercado (Adam Smith y David Ricardo, principales representantes y fundadores de esta teoría).

No obstante, es después de la segunda Guerra Mundial (19411945) cuando las ideas del desarrollo empiezan a adquirir su mayor fuerza y a adoptarse en los países, llamados desde entonces, subdesarrollados o del Tercer Mundo. 
En estas circunstancias se introduce en Colombia el concepto de desarrollo, concretamente en el año 1949 con la llegada al país de una misión económica organizada por el Banco Internacional para la Reconstrucción y el Desarrollo (BIRF, hoy Banco Mundial), liderada por el prestigioso economista canadiense Lauchlin $\mathrm{Cu}-$ rrie, para que formulara un programa de desarrollo; de esta visita se derivaron sus recomendaciones (Bases para un Programa de Desarrollo en Colombia, 1950) y el proceso de modernización e institucionalización del desarrollo (Escobar, 1988; Sánchez, 1984; López, 1995; Ocampo, 1992; Kalmanovitz, 2001).

\section{Controversia conceptual}

El concepto de desarrollo, como se sabe, estuvo asociado en un primer momento con el crecimiento económico. Más tarde, dicho concepto se asimiló con el de desarrollo económico, en el entendido de que un proceso de cambio estructural no sólo implica crecimiento sino también desplazamiento de recursos, cambio de costumbres, hábitos, actitud y posesión de bienes.

En la actualidad, el concepto de desarrollo abarca no sólo lo económico sino también lo cultural, social, político y ambiental, lo que da lugar a una gama de definiciones que varían según las teorías y escuelas del pensamiento que lo aborden, entre las cuales podemos destacar, por ejemplo, la relacionada con la globalización, considerada por algunos autores (Hernández, 2004; Romero, 2003; Ramos, s.f.; Romero, 2000) como una continuación de la modernidad que se inició, como ya se dijo, en el siglo XVIII y que hoy se ha extendido como la forma peculiar en que tiene lugar la expansión del capital, el desarrollo desigual y polarizado, el intercambio desigual, las relaciones de dominación, la exclusión, las presiones medioambientales, el incremento de la desocupación y, en consecuencia, la pobreza y la marginalidad.

Para estos autores, el concepto de globalización ha cobrado fuerza en los últimos años como consecuencia del desmantelamiento del socialismo en Europa y la desintegración de la Unión Soviética en 1989, coyuntura que aprovecharon como argumento algunos ideólogos defensores de la economía de mercado para significar el fin ideológico de la humanidad y la primacía irreversible del capitalismo como única forma para lograr el desarrollo y el bienestar (Fukuyama, 1992), en contraste con quienes entienden la globalización como una fase superior y aislada de la modernidad, denominada posmodernidad, cuyas características 
principales se basan en el estudio de los fenómenos culturales contemporáneos, como los cambios de las costumbres, creencias, concepciones, prácticas y agrupamientos sociales -identidad-propiciados por la revolución tecnológica de la informática, la biotecnología, la robótica y las comunicaciones.

\section{Las políticas regionales y sus fases de transición}

Bien se sabe que la finalidad de toda política regional -de acuerdo con la literatura existente- es disminuir las disparidades entre las regiones. En consecuencia, la variación de sus definiciones depende del enfoque que se asuma para lograrlo. En este sentido, R. Pujadas y Jaume Font, así como M. Pólese (citados por Moncayo, 2002) afirman que la política regional encierra dos connotaciones no excluyentes entre sí: a) el conjunto de medidas destinadas a reducir los desequilibrios o disparidades interregionales y b) los esfuerzos al interior de las regiones, consideradas individualmente, para superar problemas de atraso o declive económico. Esta concepción a su vez encierra sendas corrientes de pensamiento cuya transición se manifiesta en épocas y espacios geográficos diferentes, circunstancias que determinan su clasificación y denominación en el ranking de esta actividad.

Si bien no existe una clasificación universal comúnmente aceptada sobre este particular, se encuentra que algunos autores como Cuadrado (1988), Helmsing (1999), De Mattos (2000), entre otros, adoptan las siguientes fases de transición con base en criterios cronológicos y afinidades teóricas, las cuales utilizamos para efectos de este trabajo

En un primer momento (en las décadas de los cincuenta y sesenta), primaron las políticas de los modelos keynesiano y neokeynesiano -llamadas también de primera generación- en las que se distinguían dos tipos de corrientes teóricas: la que postula que el nivel de desarrollo de una región está condicionado por el posicionamiento que dicha región tenga en el sistema regional general, y la otra que hace énfasis en las condiciones internas de la región. La primera se sustenta en las teorías y enfoques del centro-periferia y de la dependencia, de la causación circular acumulativa de Myrdal y Hirschman; la teoría de los polos de crecimiento, de Perroux; y los modelos de Harrod, Domar y Kaldor, entre otros (citados por De Mattos, 2000).

La segunda, por su parte, se apoya en las teorías de Rostow, Fisher y Clark, citados por Moncayo (2002). 
Todas estas políticas tienen un aspecto en común: considerar la intervención directa del Estado en la reducción de las desigualdades interregionales, mediante mecanismos y estrategias de orden fiscal, financiero y monetario, tendentes a facilitar las condiciones favorables para la inversión privada y la acumulación del capital en las regiones de menor desarrollo (obras de infraestructura, servicios públicos, subsidios, incentivos tributarios, etcétera).

Para el desarrollo de tales políticas, en algunos países de Europa y de América Latina (Perú, México, Brasil, Uruguay y Colombia) se crearon entidades autónomas con funciones de planeación regional, con base en la experiencia de la Autoridad de los Valles del Tennessee (1933), teniendo como instrumento, hasta la década de los setenta, el modelo de los polos de desarrollo y las cuencas hidrográficas como unidades geográficas objeto de la misma (Boisier, 1994).

En un segundo momento, que comprende el periodo 19701990, ante la crisis y los cuestionamientos al modelo keynesiano, aparecen las políticas del modelo neoliberal -denominadas de segunda generación- las cuales se sustentan en las teorías de la escuela neoclásica, principalmente sobre la famosa ley de los rendimientos marginales decrecientes y los modelos de crecimiento de Solow (1956), Swan (1956). Sus postulados se basan en el libre juego del mercado, al cual se le atribuye la capacidad de resolver por sí solo los problemas de los desequilibrios interregionales, los cuales, según sostiene, se solucionan como resultado de la libre movilidad de los factores de producción (capital y trabajo).

Finalmente, desde la década de los noventa hasta la fecha, ante la crisis y los cuestionamientos al modelo neoclásico tradicional, surge una nueva vertiente conocida como teoría del crecimiento endógeno -llamada también de tercera generación-, según la cual el crecimiento económico depende de la acumulación de capital físico, capital humano y conocimiento, cuyo alcance está determinado por factores endógenos, y no exógenos como lo plantea el modelo tradicional.

De acuerdo con A. Helmsing (1999: 34), "las políticas de tercera generación enfatizan la competitividad sistémica, mientras las políticas de segunda generación se orientan hacia las acciones de las firmas y la cooperación entre las firmas; así, las políticas de tercera generación enfatizan la importancia de las condiciones básicas. Estas últimas no sólo se refieren al marco 
macroeconómico, sino también a un conjunto de acciones del nivel meso (sectorial y local), reforzando la competitividad de los sistemas regionales de producción (SRP)". Los instrumentos de estas políticas son el cluster, el distrito industrial y las cadenas productivas.

\section{Balance de la política regional keynesiana o de primera generación en Colombia}

La aparición de este tipo de políticas en Colombia, como parte integral de un plan nacional de desarrollo, ocurre a través del Plan Decenal de Desarrollo, 1960-1970, entre las cuales se destacó la de la Reforma Agraria (Leyes 135 de 1961 y $1^{\text {a }}$ de 1968) y el enfoque de los polos de desarrollo. En este periodo sobresale la elaboración, por primera vez, de un esquema de regionalización nacional, mediante el cual se pretendía ordenar el territorio a través de la localización del equipamiento urbano y las inversiones privadas; la creación de las áreas metropolitanas y oficinas de planeación municipales y departamentales (DNP, 1970). Asimismo, se multiplicaron y fortalecieron las Corporaciones Autónomas Regionales (CVC, 1954): la Corporación Autónoma Regional del Urabá (1958), la Corporación Autónoma Regional de los Valles del Magdalena y Sinú (CvM, 1960, más tarde, en 1973, Cvs, con jurisdicción en los Departamentos de Córdoba y Sucre) (DNP, 1989).

Posteriormente, en el periodo 1970-1974, en el Plan las cuatro estrategias, a cargo del economista Lauchlin Currie, bajo el enfoque del sector líder de la construcción, la política urbana se convirtió en el eje del desarrollo nacional, desplazando los problemas del sector agrario mediante el desmonte de la reforma agraria y el fomento de la migración rural urbana. Como consecuencia de ello, se introducen nuevos requisitos y condiciones para evaluar y calificar las tierras susceptibles de afectación y expropiación que se hacían prácticamente imposibles de cumplir, con modificaciones en el sistema de financiamiento (Leyes $4^{\mathrm{a}}$ y $5^{\mathrm{a}}$ de 1973 o Pacto de Chicoral), base sobre la cual se promulgó la Ley $6^{a}$ de 1975, o Ley de aparcería, y luego el programa de Desarrollo Rural Integrado (DRI), durante el gobierno liberal de Alfonso López Michelsen (Berry, 2001; Machado, 2002; Rodríguez, 2004).

Algunos autores sostienen que si bien en el periodo 19711974, años en los cuales prácticamente se paralizó el programa 
de la reforma agraria, se realizaron cambios importantes en la estructura económica (crecimiento del PIB de 5.9\% en promedio, infraestructura financiera de las Corporaciones de Ahorro y Vivienda y el sistema UPAC como mecanismo de fomento del ahorro), en lo social la inflación se disparó 7.2\% en 1970, 12.6\% en $1971,14.1 \%$ en $1972,22.1 \%$ en 1973 y $25.2 \%$ en 1974; el desempleo urbano aumentó de 9.7\% a 11.6\% (Moncayo, 2002: 13).

Los objetivos y metas propuestas en la industria de la construcción no se cumplieron -pues su ejecución se desvió hacia sectores de ingresos medios y altos, y no a la construcción de vivienda para sectores de menores ingresos como estaba previsto-, lo cual afectó la dinámica de la demanda agregada y el crecimiento del mercado interno, siendo el sector industrial el que más aportó al PIB.

Durante los años 1974-1978, si bien se intentó poner en práctica las políticas neoliberales, en realidad se continuaron aplicando los instrumentos de las políticas anteriores a través de incentivos tributarios para la desconcentración y localización de las industrias en las ciudades intermedias, teniendo como base el posicionamiento de dichas ciudades en la jerarquía del sistema nacional regionalizado, como ya se mencionó. Así, se implementa el Programa de Desarrollo Rural Integrado (DRI) como instrumento de control de la migración rural urbana y de disminución de las disparidades entre la ciudad y el campo.

En 1978-1982, cuando se empiezan a sentir las consecuencias de la deuda externa y la crisis de la economía mundial desatada a raíz del aumento de los precios del petróleo en 1973, se comienza a discutir sobre la conveniencia de una reforma constitucional (1977) encaminada a modernizar la administración del territorio nacional, donde se pone de manifiesto el tema de la descentralización administrativa

Esta situación, según Gutiérrez (2002), obligó a contratar un estudio (Bird, 1981) cuyos resultados recomiendan que se descentralicen ciertas funciones y competencias en los municipios. Recomendaciones que finalmente acogieron los gobiernos posteriores a Betancur (1982-1986) y Barco (1986-1990).

Cabe resaltar que en este periodo se inicia el proceso de privatización de algunas empresas estatales -proceso que aún continúa-, otras se fusionaron o desaparecieron en tanto que surgieron nuevas entidades e instancias de coordinación e integración regional, como los Consejos Regionales de Planificación (Corpes, 76 de 1985); al tiempo que se reconoce la importancia de la pla- 
neación urbana como instrumento de organización (9 de 1989 de reforma urbana) de la ciudad y del desarrollo urbano.

\section{Balance de las políticas regionales de segunda y tercera generación en Colombia}

Como consecuencia de las políticas neoliberales o de segunda y tercera generación -cuyos instrumentos y medidas se encaminaron hacia la internacionalización y modernización del sector agropecuario, por una parte (Ley 101 de 1993, o Ley General de Desarrollo Agropecuario y Pesquero) y, por otra, la Ley 160 de 1994 que regula la intervención del Estado y los procedimientos de la distribución y tenencia de la tierra-, la política de reforma agraria quedó relegada a crear y consolidar las condiciones del mercado de la tierra entre campesinos y terratenientes, otorgando subsidios ya no a la oferta como se venía haciendo bajo la modalidad de adjudicaciones directas, precios especiales de venta y tasas de interés preferenciales, sino a la demanda, mediante la donación de dinero o crédito a los campesinos por intermedio del sistema financiero

Con estas medidas no sólo se congelaron los programas de reforma agraria, sino que se retrocedió, ya que por medio de ellas se propició la pauperización en el campo y la migración rural urbana, beneficiando sobre todo a los terratenientes, industriales y grandes inversionistas, quienes atraídos por los incentivos y ventajas otorgadas para el cultivo de ciertos productos comerciales, como es el caso de la palma africana en la región de Urabá y el Darién (departamentos de Antioquia y Chocó), así como por las expectativas generadas por la construcción de algunas obras de infraestructura y ciertos proyectos estratégicos (vías, puertos, extracción de minerales o hidrocarburos, hidroeléctricas, entre otros), por un lado, y por el afán de acumulación de capital de algunas personas mediante los cultivos ilícitos (narcotraficantes) y el dominio territorial de grupos armados ilegales, por el otro, los campesinos terminaron siendo víctimas del conflicto, pues con frecuencia eran presionados (mediante amenazas, desplazamiento y muerte) a vender o abandonar sus tierras, en tanto que sus ocupantes o nuevos dueños eran favorecidos por los créditos y prebendas establecidos en los programas de gobierno (Contraloría General de la República, 2005).

Los efectos de estas políticas en el sector agropecuario se manifiestan también en las escasas oportunidades de trabajo, in- 
gresos y alimentación, en razón de los cambios derivados de los usos del suelo -ganadería extensiva- y de la sobrevaloración que ha tenido la tierra en las zonas donde se presentan los mayores índices de concentración de la propiedad, lo que la convierte en un recurso escaso y en un factor limitante del desarrollo rural y regional, provocando el aumento de la migración rural urbana, el alza de los precios de la canasta familiar y el deterioro de los salarios, resultado de la sobreoferta de mano de obra.

Por otra parte, entre 1990 y 1994 se eliminan las barreras arancelarias a las importaciones y subsidios a las exportaciones, al tiempo que se incentiva al sector exportador y al capital extranjero, concediéndoles beneficios de tipo fiscal, cambiario, financiero, reducción de costos laborales (Reforma Laboral, Ley 50 de 1990), privatización de empresas estatales, recorte y supresión de derechos convencionales, eliminación de la retroactividad de las cesantías, creación de los fondos privados de pensiones (Ley 100 de 1993), focalización del gasto social (SISBEN) y aumento de impuestos indirectos (IVA), entre otras (Hommes, 2002).

En los años 1994-1998, el énfasis de estas políticas recae en los problemas urbanos y el desarrollo local, fortaleciendo la planeación y los entes municipales como principales instrumentos y agentes del desarrollo (Ley 152 de 1994 o Ley Orgánica del Plan de Desarrollo, Ley 388 de 1997 o Ley de Desarrollo Territorial, la cual modifica a la Ley 9 de 1989) y se armonizan las normas y procesos de la planeación territorial con los de la planeación socioeconómica.

Desde 1998 hasta la fecha, entran en vigencia las políticas de la tercera generación a través de los nuevos criterios y enfoques establecidos para la distribución y manejo del gasto público (Ley 550 de 1999, "Por la cual se establece un régimen que promueva y facilite la reactivación empresarial y la reestructuración de los entes territoriales para asegurar la función social de las empresas y lograr el desarrollo armónico de las regiones”), así como en el concepto que se asume en la orientación del desarrollo, basada en la importancia que se le da a la educación, la investigación, la ciencia, la tecnología y la construcción de infraestructura de transporte y de comunicaciones (telefonías local, de larga distancia, celular, social, televisión, Internet, etc.). Todo ello como estrategias para aumentar la competitividad y el desarrollo, al igual que el reconocimiento que se le asigna a las pequeñas y medianas empresas (Pymes) y a la economía solidaria en el logro de estos propósitos (Ley 508 de 1999, Plan de Desarrollo 1998-2002 y Ley 812 de 
2003, Plan de Desarrollo 2002-2006), durante los gobiernos de Andrés Pastrana y Álvaro Uribe Vélez, respectivamente.

\section{Contradicciones y consecuencias de las políticas regionales en Colombia}

Si bien en la actualidad la pobreza se considera uno de los principales obstáculos del desarrollo, generadora de violencia, inconformismo, inseguridad, exclusión y descomposición social, sólo se le mira como una limitante del proceso de producción, es decir, como factor de ésta (recursos humanos) y como elemento del consumo (demanda agregada), y como tal se asume su tratamiento haciendo del gasto social no un instrumento de redistribución del ingreso con equidad -basado en las capacidades y potencialidades de las personas- sino en una modalidad de inversión del Estado cuya distribución y asignación presupuestal se hace en función de tasas de rentabilidad, relación costo-beneficio e índices de eficiencia, bajo la concepción de ver al ser humano sólo al servicio de la economía, convirtiendo la provisión de los servicios esenciales para la vida -educación, salud, nutrición, seguridad social y servicios públicos, entre otros-, que son un derecho de las personas de obligatoria responsabilidad del Estado (Constitución Política de Colombia, capítulos 2 y 5), en lucrativos negocios de particulares, y su calidad y accesibilidad -que debería estar en función de las necesidades y condiciones particulares de la gente- dependiendo de los azares del mercado.

El fundamento teórico de esta racionalidad, como se puede evidenciar hasta ahora, se expresa en el marco de las políticas ya mencionadas, mismas que si bien han contribuido a disminuir los altos índices de pobreza, como lo registran las cifras correspondientes, ${ }^{1}$ aunque de manera desigual y aun cuando en algunas regiones hayan aumentado (en Córdoba, por ejemplo), ${ }^{2}$ y que

\footnotetext{
${ }^{1}$ Según la cepal (2004), la pobreza en Colombia en el año 2002 era de 50.6\%, y según la Misión para el Diseño de Estrategias de Reducción de la Pobreza y la Desigualdad, MERPD (2004), es 52.6\%. Por su parte, los informes relativos al Índice de Desarrollo Humano (IDH) en el periodo 1990-2001, muestran que el crecimiento de dicho índice para Colombia fue de $8.5 \%$, al pasar de 0,711 a 0,771 . El índice de pobreza humana (IPH) $1990-1995$ y 1997-1999 fue de $12.8,11.1$ y 10.6, respectivamente.

${ }^{2}$ En lo que al departamento de Córdoba se refiere, el IDH para el periodo 19902001, descendió 7\%, al pasar de 0,669 a 0,622. La pobreza en el año 2004, con base en la encuesta de calidad de vida de 2003 , ascendió a $72 \%$, sólo por debajo de Chocó $(72.7 \%)$ y Boyacá $(72.5 \%)$, y por encima de la media nacional de $55.3 \%$. Según el índice de Necesidades Básicas Insatisfechas (NBI) 1997, 50\%; 2000, 44.1\%, por encima de Sucre, $48.8 \%$ y $39.8 \%$, respectivamente. En tanto que el promedio nacional NBI en el mismo periodo: $25.9 \%$ y $22.9 \%$ (PNUD-DNP, 2003).
} 
explican como hechos innegables de esta evolución el aumento de cobertura de agua potable, el mejoramiento en los indicadores nutricionales de niños menores de 5 años y el acceso a los servicios de salud. También debe decirse que dichas políticas propiciaron el desempleo y restringieron las oportunidades para el ejercicio de los derechos fundamentales a la vida, la educación, la salud, el trabajo, la seguridad social y la participación, entre otros.

Con la implementación de la Ley 715 de 2001, por ejemplo, que modifica la estructura de financiamiento a los entes territoriales y por la cual se establece un nuevo sistema de participación de los municipios en los ingresos corrientes de la nación, se contradicen los propósitos y principios de la descentralización, en la medida que su aplicación se traduce en una reducción de recursos en comparación con las cuantías previstas inicialmente en la Constitución, y en relación con la magnitud de las funciones y compromisos delegados. Pero además, con los nuevos criterios adoptados en este sistema de participación, los cuales se basan en los conceptos de la eficiencia (relación costo-beneficio) y en el esfuerzo fiscal (aumento de los recursos propios), dichos entes se ven obligados a autorizar o aumentar las tarifas de los servicios públicos, tasas, impuestos y contribuciones (predial, industria y comercio, recreación, espectáculos públicos, etc.), por una parte, y a reducir los gastos de funcionamiento -que generalmente significa recorte de personal de la administración municipal-, así como contratar con empresas particulares la provisión de dichos empleos. Propósito dentro del cual se enmarca la mencionada Ley 550 de 1999.

Así las cosas, los resultados esperados si bien se obtienen en términos de crecimiento del PIB, que es una condición necesaria pero no suficiente del desarrollo, no garantizan por sí mismos la distribución del ingreso con criterios de equidad, ${ }^{3}$ puesto que al sustentar su distribución en criterios de eficiencia y productividad, poniendo en igualdad de condiciones a todas las personas y agentes involucrados, se afecta sobre todo a la población excluida y de menores oportunidades y a aquéllos con menor capacidad de recaudo; práctica que no toma en cuenta la naturaleza desigual del desarrollo, convirtiendo dicha situación en un círculo vicioso de la pobreza cuyas consecuencias recaen principalmente en las ciudades pequeñas y medianas -que son la mayo-

\footnotetext{
${ }^{3}$ Según Libardo Sarmiento (2005), el PIB pasó de 1.9\% en 2002 a 3.5\% en 2004 y la inversión extranjera, $47 \%$ entre 2003 y 2004 . No obstante, el coeficiente de Gini se mantuvo en 0.52 en el periodo 2001-2004, ubicando al país entre los de mayor desigualdad socioeconómica en el mundo.
} 
ría-, donde las oportunidades de empleo y acceso a los servicios de salud, educación, vivienda, nutrición y otros, son más escasas, precarias y limitadas.

Esta desigualdad debería ser recompensada por el Estado concediendo, por ejemplo, más y mejores subsidios al desempleo, a la vivienda, los servicios públicos, la ampliación de beneficios y cobertura de la seguridad social, mejores salarios y estímulos tanto a la pequeña y mediana empresas como a la producción y los negocios que se sustentan en el trabajo propio, sin embargo, poco se valora debido a los fuertes intereses y al dominio que ejercen los grandes conglomerados económicos en la formulación de las políticas públicas nacionales y regionales, y también a las condiciones impuestas al gobierno nacional por los organismos de crédito internacional (FMI y BM) que se traducen en fuertes restricciones de carácter fiscal, financiero y monetario, fundamentalmente. ${ }^{4}$

\section{El caso del departamento de Córdoba}

El departamento de Córdoba, Región Caribe de Colombia, se caracteriza en el concierto de la economía mundial, nacional y regional por la inmensa riqueza de su biodiversidad $(700,000$ ha de bosque con cerca de 200 especies maderables, $130 \mathrm{~km}^{2}$ de vegetación manglar acompañada de un inmenso ecosistema fluvial y marítimo que se convierte en refugio y criadero natural de aves y peces de variadas especies); recursos mineros (reservas de carbón, oro, gas natural y ferroníquel) y por su alto potencial agrícola y ganadero, conjunto de factores que lo sitúan en una posición privilegiada.

\subsection{Evidencias y secuelas de las políticas regionales}

No obstante lo anterior, este departamento ha sido poco beneficiado por las políticas mencionadas, situación que explica, en parte, el contraste entre su enorme potencial y su actual grado de desarrollo, aspecto que constituye el propósito de este trabajo.

Una revisión empírica superficial sobre los alcances e implicaciones de estas políticas en esta zona del país, permiten identificar y diferenciar ciertos rasgos de su aparición en distintos momentos y tipos de manifestaciones.

\footnotetext{
${ }^{4}$ Véase www.banrep.gov.co. Acuerdo Stand-by de Colombia con el FMI, abril de 2005.
} 
El proyecto de la hidroeléctrica de Urrá, por ejemplo, que desde un primer momento (década de los setenta) se concibió, debatió y gestionó como un proyecto multipropósito de desarrollo regional, en su momento la clase política y la dirigencia regional lo consideraron como la panacea al subdesarrollo. Sobre este proyecto se hicieron múltiples estudios, planes y proyectos patrocinados por el gobierno central y la banca internacional (Plan Maestro de Desarrollo Integral de la Cuenca del Río Sinú, 1983), pero su aprobación finalmente se hizo por motivos energéticos de carácter sectorial (abril, 1993), es decir, bajo la óptica de las políticas neoliberales o de segunda generación del gobierno de César Gaviria, con lo cual no sólo se frustró una generación de expectativas, sino también se perdió un cúmulo de esfuerzos y recursos institucionales, sin que hasta el momento se sepa si dicha alternativa fue la más viable para mitigar o compensar los daños ambientales causados por la construcción y operación de dicho proyecto.

El proyecto minero de Cerro Matoso (1979), el cual inicia operaciones en 1982, se construye también como un proyecto sectorial de mucha importancia para el país y la región, principal fuente de divisas de Colombia y de regalías para el departamento, la Corporación Autónoma Regional de los Valles del Sinú y de San Jorge, así como los municipios de su área de influencia.

En lo que concierne al sector de la agricultura, se debe advertir que el departamento de Córdoba es de vocación esencialmente agropecuaria, la economía se sustenta en los cultivos transitorios de pancoger (yuca, ñame, plátano, arroz, ajonjolí y maíz), aunque también en los de algodón, maíz y arroz con fines comerciales. Así evolucionó hasta el año 1975, cuando se introdujo el sorgo como un cultivo promisorio, cubriendo inicialmente un área de 21,892 hectáreas hasta llegar a un total de área cultivada de 125,664 ha. Luego, en 1985, aparece el coco (3,700 ha) y un poco de cacao $(0.65 \mathrm{ha})$, para un total en ese mismo año de 141,400 hectáreas.

La producción, por su parte, registró un incremento de 54,914 toneladas (11.87\%), pasando de 462,306 en 1975 a 517,220 en 1985 y un incremento promedio en el rendimiento de 0.173 ton/ ha, que equivale a 4\%. Entre 1975 y 1985 se destacan el sorgo, con un incremento de $790 \%$ en el área cultivada y su rendimiento pasa de 2.3 a 3.2 ton/ha; el arroz, por la disminución de $-43.4 \%$ en su área cultivada y por el aumento en su rendimiento, de 3.7 a $4.8 \%$ ton/ha; el maíz, al aumentar su área cultivada en $21.83 \%$ y 
su rendimiento, pasa de 1.8 a 2.1 ton/ha; al igual que el plátano, que disminuye su área en 39\% al pasar de 28,500 en 1975 a 17,500 en 1985.

En 1995, cuando empiezan a implementarse las políticas neoliberales o de segunda generación, el área cultivada se incrementó 36,235 ha, es decir, $25.6 \%$ en relación con 1985, exactamente el doble de la registrada en dicho año. La producción se aumentó 148,494 toneladas $(28.71 \%)$ y el rendimiento promedio (ton/ha) creció 8.75\%, más del doble del producido en 1985.

Entre los cambios más significativos ocurridos entre 1985 y 1995 sobresalen: el algodón, con un incremento de 77\% en su área cultivada; el maíz, $36.5 \%$ en el área cultivada y en su rendimiento, al pasar de $2.1 \%$ a $2.3 \%$ ton/ha; la yuca, $154.5 \%$ en el área; el ñame, que pasa de 7 a 10.9 ton/ha, con un leve crecimiento de su área; el frijol, como cultivo promisorio con 742 ha; el sorgo, que disminuye su área en -48\%, lo mismo que el ajonjolí, en $-57.6 \%$, y desapareciendo el cacao y el coco.

Entre 1995 y 2004, en pleno auge de las políticas neoliberales de la competitividad, el área cultivada sólo se incrementó en 7,025 ha $(3.95 \%)$, lo que provocó la caída de la tendencia creciente desde 1975, en tanto que la producción aumentó en 315,321 ton (47.6\%) y el rendimiento en $35.85 \%$ (gráfica I).

\subsection{Comportamiento del ingreso per cápita y el empleo}

Sin pretender establecer una relación de causalidad entre el comportamiento de la producción agrícola antes anotado y los ingresos y empleos en el ámbito departamental, sino como un simple dato de referencia, se encuentra que, de acuerdo con las cifras del Departamento Nacional de Estadísticas (DANE, 2002), gráfica II, el PIB por habitante a precios constantes de 1994 del departamento de Córdoba durante el periodo 1990-2002, presenta una tendencia creciente y una variación anual superior al promedio nacional, pasando de $55.8 \%$ en 1990 a $68.1 \%$ en 1995 y $80.9 \%$ en 2002 , con una tasa anual de crecimiento de $1.33 \%$ frente a $0.93 \%$ del país, es decir, entre 1990 y $1995,1.14 \%$ y $1.04 \%$; en 1996-2002, 1.09\% para Córdoba y 0.96\% el nacional.

Con base en estos indicadores, al menos cabría esperar una tendencia decreciente del desempleo, puesto que, se supone, dicho crecimiento implica mayores oportunidades de trabajo, de comercialización y consumo de bienes y servicios. 


\section{Gráfica I}

Área cultivada, producción y rendimiento (ton/ha) de cultivos transitorios, departamento de Córdoba, 1975-2004

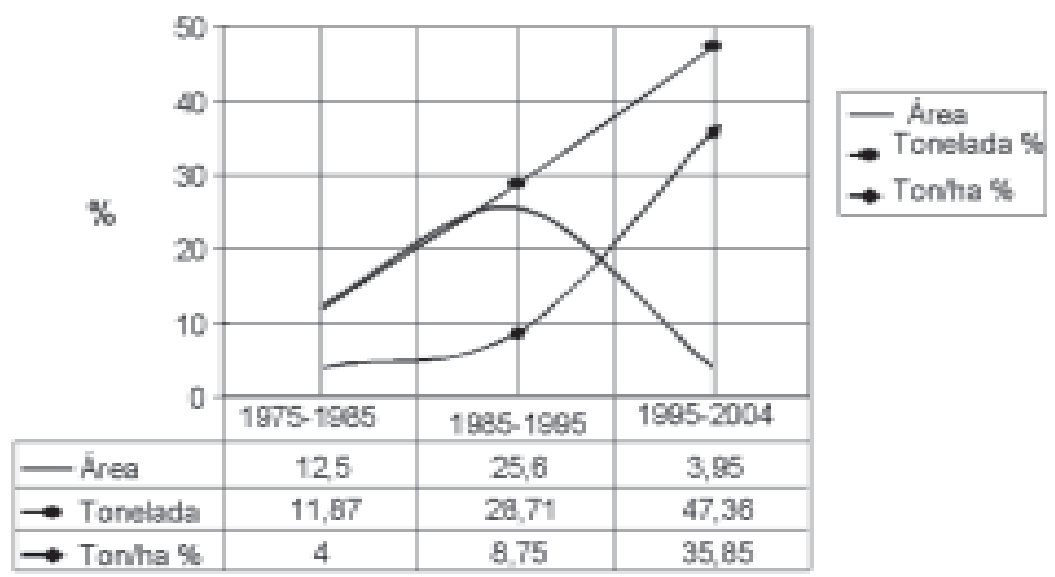

Fuente: Elaboración propia con base en DANE (1974), anuarios estadísticos del departamento de Córdoba (1976, 1977, 1982), Banco de la República, sección Córdoba (1976, 1978-1982), Cámara de Comercio de Montería (1977), Centro de Estudios de la Ganadería (1985), Ministerio de Agricultura (2004).

\section{Gráfica II}

PIB por habitante a precios constantes de 1994, departamento de Córdoba y el promedio nacional, 1990-2002

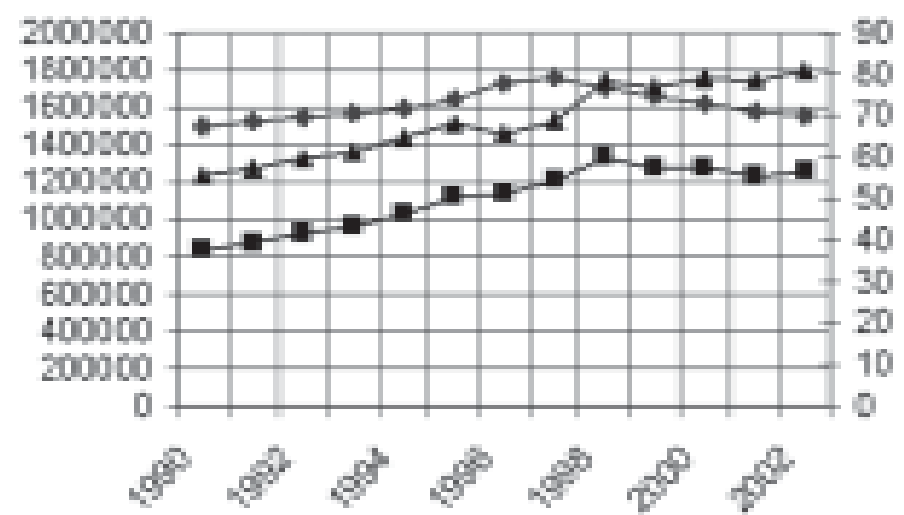

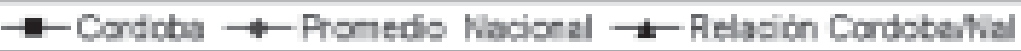

Fuente: Elaboración propia con base en las estadísticas del DANE, 2002. 


\section{Gráfica III}

Tasa de desempleo en Montería y siete ciudades colombianas, 1985-1995

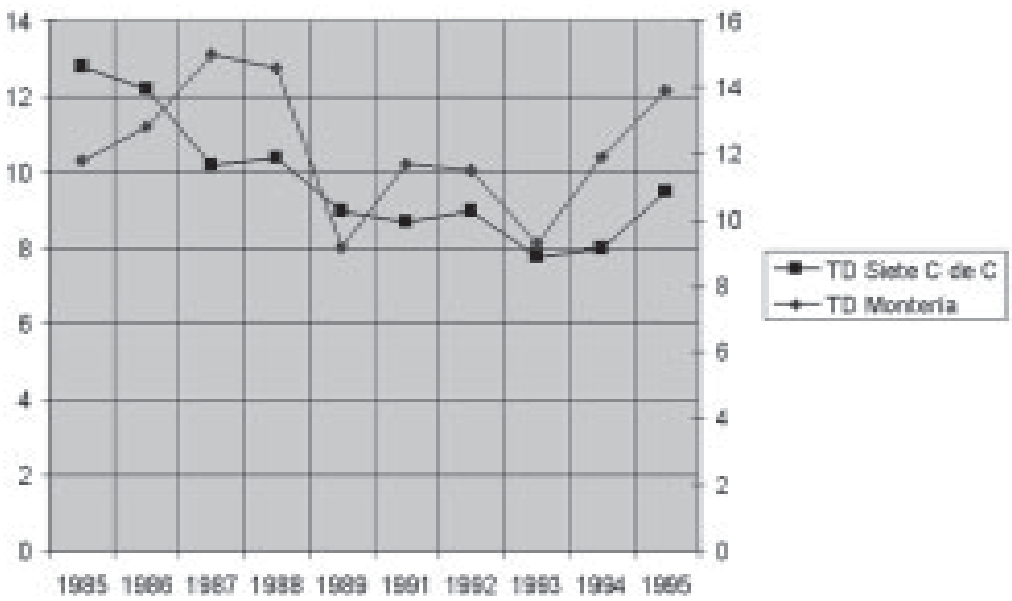

Fuente: Elaboración propia con base en las estadísticas del DANE y el Banco de la República.

Gráfica IV

Tasa de desempleo en Montería y siete ciudades colombianas, 1996-2004

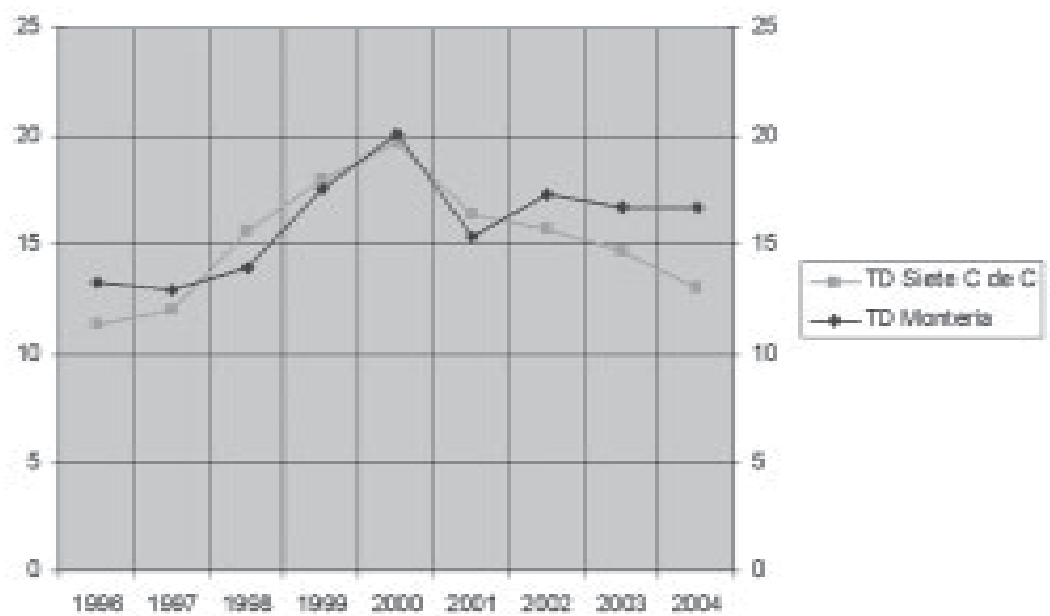

Fuente: Elaboración propia con base en las estadísticas del DANE y el Banco de la República. 
Sin embargo, si se analizan los datos del DANE (gráfica III), se observa que el desempleo en Montería, la capital del departamento, en el periodo 1985-1995 tuvo un comportamiento ascendente, excepto 1989 y 1993, con una tasa media anual de crecimiento de $12.17 \%$, inmensamente superior a la registrada para siete ciudades colombianas (9.76\%). En 1996-2004 se incrementó a $16 \%$ frente a $15.1 \%$ del promedio registrado para las mismas ciudades, con el agravante de que mientras en éstas se registra una leve tendencia decreciente a partir de 2000, en Montería sucede lo contrario (gráfica IV).

Parte de esta explicación podría estar en la pérdida que significó, en términos de empleo, la disminución de 29,310 ha de área cultivada durante el periodo 1995-2004 en relación con las de 1985-1995, lo que equivale a un número aproximado de 8,500 empleos, ${ }^{5}$ cifra que, si no la absorbieron otros sectores y ramas de la economía regional, como se presume, se constituye naturalmente en fuente de migración y desempleo.

Según el DANE, la participación del sector agropecuario en el PIB departamental pasó de $51.5 \%$ en 1985 a $32.3 \%$ en 1995 у a $26.4 \%$ en 2000; en tanto que el sector industrial lo hizo en $11.2 \%$, $12.2 \%$ y $2.1 \%$, respectivamente, de donde se infiere que gran parte de la población desplazada por el crecimiento económico pasó a engrosar el mercado de la informalidad y de los servicios. Así se puede leer en las cifras del DANE cuando indican que este sector pasó de representar $37.3 \%$ en 1985 a $55.6 \%$ en 1995 y $50 \%$ en 2000 del PIB departamental (DANE, 2000).

Asimismo se observa que, según el Informe de Coyuntura Económica Regional de Córdoba (segundo semestre de 2004), los sectores de la construcción, el transporte y las comunicaciones son los que ganaron mayor participación en informalidad al presentar variaciones de $37.6 \%$ y $10.7 \%$, al igual que el sector comercial que participa con $39.8 \%$ del total de dicha población en la ciudad de Montería, cuyo nivel de informalidad se ha incrementado de 85,704 personas en 2003 a 86,126 en 2004 (DANE, 2000: 21).

En estas circunstancias es fácil comprender el fenómeno del mototaxismo que, según encuestas y estudios realizados por distintas entidades, pasó de 1,633 motos en 2003 a 4,277 en 2006 que prestan el servicio de transporte público urbano, lo cual se

\footnotetext{
${ }^{5}$ Para tal efecto se estima un parámetro de 173 jornales por empleo, es decir: arroz, 82 jornales/ha; algodón 120 jornales/ha y sorgo 13.5 ha por empleo.
} 
ha convertido en un nuevo problema de difícil manejo y múltiples repercusiones en la ciudad. ${ }^{6}$

Paralelamente cabe señalar el auge que ha tenido la inversión en Montería en la última década, principalmente en la construcción de departamentos de lujo, centros comerciales, boutiques, hoteles y restaurantes de calidad, que no demandan gran cantidad de mano de obra ni garantizan buena remuneración, prestaciones sociales ni estabilidad laboral, situación que se ve favorecida con la implementación de las reformas laborales (Ley 50 de 1990 y la Ley 789 de 2002). Es importante aclarar que esta última extendió la jornada ordinaria de trabajo desde las 6:00 hasta las 22:00 horas, eliminando los sobresueldos nocturnos y las horas extras, lo que disminuye el valor de su remuneración a $75 \%$ del salario ordinario proporcional a las horas trabajadas y el reconocimiento de los domingos y días festivos (Gaviria, 2002).

De esta manera se establece que el crecimiento del PIB de la economía cordobesa no es el resultado de la expansión de su mercado sino, en gran parte, fruto del aumento de las exportaciones de ferroníquel, favorecido por el alza de su precio en el mercado internacional, situación que no se refleja en la creación de nuevos puestos de trabajo pese al aumento generado en las ganancias.

Según un documento del Ministerio de Comercio Exterior de Colombia (2001: 5) sobre el comportamiento de las exportaciones, el incremento de $34 \%$ de éstas en el departamento de Córdoba en 2000, en relación con las del año anterior, se explica por los mayores ingresos por la venta de ferroníquel que crecieron alrededor de $37 \%$, en especial por el alza en los precios internacionales (43.6\%), lo que lo posicionó como el artículo con mayor representatividad, con $94.7 \%$ del total del valor libre a bordo (Free on bord, FOB).

De acuerdo con el Informe de Coyuntura Económica Regional Córdoba (DANe, Banco de la República, 2003), "En el año 2002 se produjeron 96'974,769 libras de níquel, superando en 14.4\% lo emanado en 2001. En relación con el precio, éste presentó una tasa de crecimiento anual positiva de 13.7\%. Este óptimo desempeño se atribuye a que el montaje de la segunda línea instalada en años anteriores en la planta entró en una etapa de mayor estabilidad, lo que ocasionó por ende un incremento en la producción. Lo anterior coincidió con el cierre de algunas plan-

\footnotetext{
${ }^{6}$ Fundación Universitaria Luis Amigó, Centro Regional Montería, inventario de mototaxistas en Montería, febrero de 2003 y Universidad Pontificia Bolivariana, sección Montería, 2006.
} 
tas productoras de níquel en el mundo por problemas de costos y a la mayor demanda de ciertas empresas metalmecánicas del mineral" (DANE-Banco de la República, 2003: 29).

En el primer semestre de 2004 (DANE, 2004) se produjeron 53'968,520 libras de ferroníquel, lo que incrementó la producción en $5.2 \%$ en relación con la misma etapa de 2003 . Respecto del precio promedio del mineral en la Bolsa de Londres, se mantuvieron altas cotizaciones del mismo a lo largo del periodo de análisis, generando una tasa de crecimiento anual de 63.1\%, el cual se atribuye a la alta demanda de acero por parte de los países asiáticos, principalmente de China.

\subsection{Violencia, desplazamiento y tenencia de la tierra}

Según la Consultoría para los Derechos Humanos y el Desplazamiento (Codhes) y la Conferencia Episcopal de la Iglesia Católica, en el periodo 1985-1998 el número de personas desplazadas en Colombia fue de 1'535,000; 2'914,853 en 2002 y 3'800,000 para junio de 2006. Asimismo se señala que este movimiento de personas se registró en 220 municipios y 27 departamentos del país, y apunta los siguientes motivos: amenazas 46\%, homicidios $17 \%$, atentados $11 \%$, desalojos $6 \%$, torturas $5 \%$, bombardeos $2 \%$, secuestro, extorsión y boleteo, $1.11 \%$. $^{7}$

En un estudio sobre el desplazamiento interno forzado en Colombia y apoyado en un informe de la Contraloría General de la República del 10 de octubre de 2000, Édgar Forero (2003: 5) estima que entre 1985 y 1996 los predios mayores de 500 ha pasaron de 9.6 millones a 19.2 millones de ha, en tanto que las propiedades menores de 5 ha pasaron de 3.4 millones en 1985 a 2.2 millones de ha en 2001, siendo las regiones Norte de Santander, Urabá Antioqueño y Magdalena Medio donde se dieron los mayores índices de concentración.

En lo que concierne al departamento de Córdoba, el histórico fenómeno de la migración -hoy desplazamiento forzoso- está muy asociado con la concentración de la tenencia de la tierra y la permanente inestabilidad de las políticas de reforma agraria; misma que se inició desde el año 1944 (Ley 100), cuando despareció la Ley 200 de 1936, y que originó las luchas campesinas y la violencia rural en distintas partes del territorio nacional, sien-

7 www.Codhes.org; El Espectador, 11/07/00, p. 3B; El Tiempo, 14/09/03, pp. 126; El Tiempo, 04/09/03, pp. 1-2; El Tiempo, 12/09/06, pp. 1-2; El Tiempo, 17/09/06, pp. 1, 22-23. 
do precisamente el departamento de Córdoba una de las regiones donde se registró la mayor parte de este tipo de experiencias, entre las que destacan las luchas campesinas de Loma Grande (1930), las de Cispatá-Tinajones (1960) y, en la década de los setenta, las de Chuhurubí (Cereté), El Cerrito (Montería), Arroyón (Planeta Rica) y Corinto (Ayapel) (Exbrayat, 1971; Sánchez, 1975; Kalmanovitz, 1978; Fajardo, 1986; Fals Borda, 1982).

De acuerdo con cifras del Censo Nacional Agropecuario de 1970, las explotaciones en terrenos menores de cinco hectáreas incrementaron su participación dentro del total departamental, pasando de $59.2 \%$ en 1960 a $61.4 \%$ en el 1970 , en tanto que la superficie descendió de $2.2 \%$ a 1.6\%. Las explotaciones, así como la superficie bajo la dirección del productor, descendieron de $97.7 \%$ a $92.8 \%$ y de 76.15 a $60.2 \%$, respectivamente.

En 1984, según datos del Centro de Estudios de la Ganadería (CEGA, 1985), 95\% de los predios menores de cinco hectáreas en el departamento de Córdoba ocupaban solamente $0.02 \%$ de la superficie, mientras que a $0.08 \%$ de los predios mayores de 100 hectáreas les corresponde 59\% de la misma (CEGA, 1985).

Estudios de la Consultoría en Derechos Humanos y el Desplazamiento (Codhes, 1996) señalan que la ciudad de Montería, capital del departamento de Córdoba, en la segunda mitad de la década de 1980 concentró poblaciones desplazadas por violencia provenientes del Alto Sinú y San Jorge y de familias damnificadas por el invierno que se ubicaron en condiciones de pobreza en barrios marginales densamente poblados (Codhes, 1996). ${ }^{8}$

En el mismo informe también se menciona que desde 1995 una nueva ola migratoria hacia el departamento de Córdoba se hizo evidente como consecuencia de las acciones armadas de grupos paramilitares, fuerza pública y guerrilla en la zona de Urabá, la cual adquirió dimensiones alarmantes por los asesinatos y masacres que generaron el más grande éxodo de los últimos años.

Para comprender este fenómeno, un tanto extraño y difícil de entender, hay que ir a los antecedentes y características del conflicto colombiano, ${ }^{9}$ según los cuales en el Urabá, región tradicionalmente bananera y estratégica del país por su posición geopo-

\footnotetext{
${ }^{8}$ Para mayor información también se puede consultar: Fundación Internacional de Ayuda Humanitaria contra el hambre (2002), "El desplazamiento por la violencia en Córdoba 1999-2002", en www.disaster-info-net/desplazados.

${ }_{9}$ Para mayor información, consultar www.cinep.org, "Deuda con la humanidad. Paramilitarismo de Estado en Colombia”.
} 
lítica, convergieron, y aún existen, grupos guerrilleros de distintas ideologías de las Fuerzas Armadas Revolucionarias de Colombia (FARC), el Ejército Popular de liberación (EPL), el Ejército Revolucionario del Pueblo (ERP), el Ejército de Liberación Nacional (ELN) y paramilitares. Entre 1991 y 1994 se desmovilizó el EPL, grupo paramilitar liderado por Fidel Castaño, el ERP y la Corriente de Renovación Socialista (grupo disidente del ELN). Previo a ello, en 1988, se da la primera incursión de las Autodefensas en el departamento de Córdoba a través de la masacre de "Mejor esquina", corregimiento del municipio de Buenavista. En tanto que en el Urabá ocurrieron las masacres de la "Chinita" y la "Hondura", donde las víctimas fueron en su mayoría obreros de las bananeras, afiliados al sindicato agrario, supuestamente simpatizantes y colaboradores de la guerrilla.

Entre 1994-1996, la guerrilla de las FARC no sólo ocupó los territorios abandonados por las guerrillas desmovilizadas, sino que también persiguió y aniquiló a una fracción de desmovilizados del EPL (Masacre "La Negra", Apartadó) por supuesta colaboración con la fuerza pública y los grupos paramilitares, de donde se originó la conformación de las Autodefensas Campesinas de Córdoba y Urabá (ACCU) y más tarde las Autodefensas Unidas de Colombia (AUC), desatándose así la espiral de violencia, masacres y desplazamiento que aún persiste.

Por las recientes confesiones del líder paramilitar Salvatore Mancuso, se sabe que las empresas multinacionales bananeras del Urabá financiaron y colaboraron con grupos paramilitares establecidos en dicha zona, al igual que varios generales y miembros del ejército nacional. ${ }^{10}$

Un estudio más reciente de la Contraloría General de la República (2005) estima que el desplazamiento de los campesinos y sus familias, así como el despojo de sus propiedades en los últimos cinco años ha afectado 265 mil hogares que, de acuerdo con los cálculos de la Contraloría, poseían más de 2.6 millones de hectáreas, con un valor aproximado de 6.1 billones, siendo los departamentos más afectados, en orden de importancia: Antioquia, Caquetá, Cesar, Chocó, Magdalena, Bolívar y Córdoba. Paradójicamente, "mientras el Instituto Colombiano de la Reforma Agraria (Incora, hoy Incoder) en cuarenta y dos años distribuyó 1.8 millones de hectáreas dentro de la frontera agrícola, puesto que el resto de su labor fue la adjudicación de tierras baldías, los grupos ilegales, en apenas cinco años, han auspiciado

${ }^{10}$ Revista Cambio, 725, mayo de 2007, en: www.revistacambio.com.co. 
el despojo de tierras a los campesinos en mucho más que eso" (Contraloría General de la República, 2005).

\section{Síntesis y conclusiones}

La situación antes analizada y su relación con los fundamentos, la estructura, el objeto de intervención y la finalidad de la política regional, permite establecer las siguientes conclusiones.

Los instrumentos y medidas de política regional predominantes en Colombia se aproximan más al modelo de la segunda y tercera generación, con una incipiente influencia de ésta última, es decir, el modelo de crecimiento endógeno.

En términos generales, la estructura de dichos instrumentos se compone de dos niveles: uno de carácter global, en poder del gobierno central, mediante el cual se asignan los recursos productivos (tierra, crédito, tecnología) y la redistribución del ingreso y gastos en las regiones.

El otro, de carácter micro, bajo el poder de los gobiernos locales, por medio de los cuales se establece el diseño, se fomenta la inversión privada en las respectivas localidades, se coordina con las instituciones en los ámbitos local y departamental y se asignan los recursos físicos, humanos y financieros. Disponiendo para ello de los recursos transferidos por el gobierno central, de acuerdo con las normas constitucionales y legales vigentes. En tal sentido, el gasto público de la inversión municipal se concentra en la construcción de obras de infraestructura, provisión de servicios y capacitación de la mano de obra (educación básica, técnica y tecnológica), fundamentalmente para atraer capital y apoyar la producción.

En este tipo de estructura -que si bien es consecuente con el modelo neoliberal y las características de la economía globalizada, cuya estructura se configura en redes o nodos de pequeñas y medianas empresas localizadas en distintos lugares del espacio geográfico (Castells, 1999)- las transacciones y procesos productivos, en aras de la competitividad, exigen estructuras horizontales, ágiles y flexibles, sin embargo, adolece de una instancia intermedia que coordine y concerte con el ámbito central los propósitos y prioridades de dichas políticas, dadas precisamente las disparidades y particularidades de las regiones, como se establece en la Constitución Política (artículos 306 y 307) y se espera que se reglamente en la Ley de Ordenamiento Territorial, actualmente en discusión en el Congreso de la República. 
Dicha falencia, en lugar de contribuir al fortalecimiento de una red diversificada de ciudades, que propicie el desarrollo local según las potencialidades y particularidades regionales, lo que hace es propiciar el aislamiento y fragmentación de éstas, pues se observa la concentración de las actividades económicas y el equipamiento urbano en las áreas metropolitanas y capitales de departamentos, como en el caso de Montería y Córdoba donde, además de los problemas señalados a lo largo de este estudio, también empieza a sentirse un nuevo fenómeno: el desplazamiento interno y la segregación social y físico-espacial en la zona urbana, que en nada favorece la consolidación de su estructura, sino que, por el contrario, lo que se observa es una gama de emplazamientos sin equipamiento y con pésimos servicios públicos, y ni qué decir de la calidad de sus viviendas.

No obstante, otra razón que contribuye a este problema, pero también ayuda a su explicación, es el carácter regresivo y de inequidad que se implantó a través de la Ley 715 de 2001, que reformó el sistema de transferencias estipulados en la Constitución Política de 1991 (artículos 356 y 357, reglamentados por la Ley 60 de 1993), donde se decía (art. 357) que los recursos serían distribuidos de conformidad con los siguientes criterios: $60 \%$ en proporción directa al número de habitantes con necesidades básicas insatisfechas y al nivel relativo de pobreza de la población del respectivo municipio; el resto en función de la población total, la eficiencia fiscal y administrativa y el progreso demostrado en calidad de vida, asignando en forma exclusiva un porcentaje de esta parte a los municipios menores de 50,000 habitantes, y que dichos recursos tendrían una participación que aumentaría anualmente de $14 \%$ de los ingresos corrientes de la nación en 1993, a 22\% en el año 2002.

En la actualidad, la participación para educación, por ejemplo, en virtud de la mencionada Ley 715 , se realiza según criterios de población atendida, población por atender y un indicador de pobreza certificado por el DANE. El valor de la transferencia para una entidad territorial se determina estimando el costo por estudiante en el sistema educativo y multiplicándolo por la población beneficiaria de la entidad territorial. Si quedan recursos después de la transferencia, éstos serán distribuidos según los criterios de población potencial y de equidad.

Desde este punto de vista, se desconoce el carácter desigual de las regiones y se favorece a los departamentos y municipios con mayor porcentaje de cobertura, como concluyen J. Barón y 
A. Meisel en su estudio sobre la descentralización y las disparidades económicas regionales en Colombia en la década de los noventa: "Las economías departamentales más diversificadas, en especial con una mayor base industrial, y de mayores ingresos, son las que tienen mayor participación de los recaudos locales como proporción del PIB. Así, estos departamentos enfrentan menos restricciones en el uso que le pueden dar a sus ingresos propios comparados con aquellos departamentos que dependen en gran medida de las transferencias (condicionadas) de la nación” (Barón y Meisel, 2003: 38).

La misma situación se presenta para el caso de la salud, con el agravante de que en este tema los criterios adoptados están en función de garantizar el mercado de la salud, financiando ya no a la oferta, como lo establecía el sistema anterior, sino a la demanda.

$\mathrm{Al}$ respecto, se comprueba que los recursos transferidos para este sector tienen tres destinos específicos: 1) financiamiento de subsidios a la demanda, 2) prestación de servicios de salud a la población pobre en la porción no cubierta con los subsidios a la demanda y 3) acciones de salud pública.

Si bien existen criterios de estratificación socioeconómica y del sistema de identificación de beneficiarios de programas sociales (Sisben) en los municipios, para focalizar y distribuir el gasto social y para otorgar subsidios para la salud, la educación y otros servicios públicos, se constata, en el caso de la salud, la manipulación y corrupción en su manejo, principalmente en aquellos municipios y departamentos donde los grupos armados al margen de la ley mantienen el control del territorio, de lo que no es ajeno el departamento de Córdoba y otros de la Costa Caribe de Colombia.

Por lo anterior, podemos concluir que los instrumentos de la política regional de carácter redistributivo en Colombia, en nada favorecen la disminución de las disparidades regionales ni la pobreza de los municipios de menor grado de desarrollo, puesto que su distribución es directamente proporcional a la riqueza y capacidad de gestión de los entes territoriales e inversa a su pobreza y débil gestión, con el agravante que los obliga a competir entre sí por la inversión en infraestructura y equipamiento que hagan atractiva la inversión extranjera en detrimento de la inversión social y el desarrollo de capacidades humanas.

Pero, además, con la implantación de estas medidas de política regional prácticamente se desmonta en Colombia el modelo 
de descentralización concebido en la Constitución Política de 1991, y convierten a los entes territoriales en simples entidades ejecutoras de los programas del gobierno central de turno, sin autonomía y sin poder político de negociación.

En consecuencia, no basta exigir un cambio de política regional, también es necesario empezar a construirla desde el ámbito local sobre la base de las oportunidades que brinda la globalización y lo que aún queda del régimen de descentralización, en el entendido de que el éxito de dicha política se sustenta fundamentalmente en el fortalecimiento del tejido social de las localidades a partir y en función de las satisfacción de sus necesidades. Lo cual implicará una política integral y articulada al desarrollo humano, cuyos instrumentos de asignación y distribución de recursos, ingresos y gastos se hagan preferentemente con criterios de equidad, fomentando la participación ciudadana no sólo en la planeación, ejecución y control por separado, sino en todas ellas al mismo tiempo.

Una política de esta naturaleza deberá sustentarse en una concepción de la economía y del desarrollo en general, según la cual todos los procesos sociales son contradictorios y desiguales y que sólo esa desigualdad es la que los mantiene y dinamiza.

Bajo esta óptica, la búsqueda del desarrollo y la disminución de la pobreza sólo será posible si se integran todas las fuerzas sociales y económicas, pero respetando la diversidad de intereses, concepciones, costumbres y cultura de la gente. Protegiendo y ayudando al más débil, pero aprovechando sus fortalezas, brindando y ampliando oportunidades no sólo en función del crecimiento económico, sino también como una condición previa para lograrlo.

En ese sentido, la competitividad que hoy día está de moda será verdaderamente sistémica, más fuerte y sostenible.

\section{Bibliografía}

Acuerdo Stand-by de Colombia con el FMI (2005), www.banrep.gov.co, abril de 2005.

Barón, R., Juan D. y Adolfo Meisel-Roca (2003), "La descentralización y las disparidades económicas regionales en Colombia en la década de 1990", Documentos de Trabajo sobre Economía Regional, 36, Centro de Investigaciones Económicas del Caribe Colombiano-Banco de la República Cartagena de Indias, en www.banrep.gov.co, pp. 1-82. 
Berry, Albert (2001), “¿Colombia encontró por fin una Reforma Agraria que funcione?, Revista de Economía Institucional, 4 (6), Departamento de Economía, Universidad de Toronto, Canadá, pp. 24-70.

Bird, Richard (1981), Finanzas intergubernamentales en Colombia, Informe final, Bogotá, Departamento Nacional de Planeación.

Boisier, Sergio (1994), "Crisis y alternativas en los procesos de regionalización”, Revista de la CEPAL, 52, Santiago de Chile, pp. 179-189.

Castells, Manuel (1999), “Globalización, sociedad y política en la era de la información” Revista análisis político, 37, Instituto de Estudios Políticos y Relaciones Internacionales Universidad Nacional de Colombia, Bogotá, Colombia, pp. 3-16.

Castoriadis, Cornelius (1991), "Reflexiones sobre el 'desarrollo' y la 'racionalidad', en Fernando Viviescas y Fabio Giraldo (comps.), Colombia, el despertar de la modernidad, Bogotá, Carvajal.

CEgA (Centro de Estudios de la Ganadería) (1985), Distribución de la propiedad rural en Colombia, 1960-1984”, Bogotá, CEGA.

Codhes (Consultoría de Derechos Humanos) (1996), Un país que buye, Bogotá, Codhes.

Contraloría General de la República (2005), “Territorio, patrimonio y desplazamiento: insuficiencia y deficiencia de las políticas estatales", Intervención del Vicecontralor General de la República, Luis Bernardo Flórez Enciso, en el evento de constitución de la Mesa de Tierras convocado por la Procuraduría General de la Nación, Bogotá D.E., Hotel Tequendama, Agosto 30 de 2006 en www.contraloriagen. gov.co:8081/.../2625/

Contraloría General de la República (2005), "El desplazamiento forzoso de los colombianos y sus impactos patrimoniales", en <www.banrep.gov.co>, 18 de abril. 
Coraggio, José Luis (1977), "Posibilidades y dificultades de un análisis espacial contestartario", Revista Demografía y Economía, vol. XI, 2 (32), México.

Coraggio, José Luis (1979), "Sobre la especialidad social y el concepto de región", Avances de Investigación no. 3, COLMEX, México.

Coraggio, José Luis (2001), "La promoción del desarrollo económico local en las ciudades: el rol de los gobiernos municipales”, ponencia presentada en la Reunión Anual de trabajo en red núm. 5, Montevideo, 31 de mayo.

Cuadrado-Roura, Juan R. (1988), "Políticas regionales hacia un nuevo enfoque", Papeles de Economía Española, 35, en http://dialnet.unirioja.es, pp. 68-95.

CEPAl (Comisión Económica para América Latina y el Caribe) (2004), Panorama Social de América Latina, capítulo I Pobreza y distribución del Ingreso en http://www.eclac.cl/ publica ciones/xml/6/20386/PSE2004_Cap1_Pobreza.pdf p 62.

DANE (Departamento Nacional de Estadísticas) (1960), Censo Nacional Agropecuario, Medellín, Bogotá, DANE.

DANE (Departamento Nacional de Estadísticas) (2000), Cuentas regionales, Bogotá, en <www.dane. gov.co $>$.

DANE (Departamento Nacional de Estadísticas) (2002), Cuentas departamentales-Colombia, PIB departamental por habitante a precios constantes de 1994, periodo 1990-2002, Bogotá, en <www.dane.gov.co>.

DANE-Banco de la República (2003), Informe de coyuntura económica regional de Córdoba, primer semestre, Bogotá, en $<$ www.dane.gov.co $>$.

DNP, (1970), "Políticas de desarrollo regional y urbano modelo de regiolanización”, Revista de Planeación y Desarrollo, II (3), Bogotá, pp. 303-352. 
DNP (1989), "Anotaciones sobre las corporaciones autónomas regionales, síntesis de su gestión en 36 años de existencia”, Revista de las Corporaciones Regionales, 1, Bogotá, pp. 97-103.

Escobar, Arturo (1988), "La profesionalización e institucionalización del 'desarrollo' en Colombia durante el periodo inmediatamente posterior a la segunda Guerra Mundial”, Revista Universidad de Antioquia, 214, Medellín, Colombia, pp. 4-43.

Exbrayat, Jaime (1971), Historia de Montería, Montería, Imprenta Departamental.

Fajardo, Darío (1986), Haciendas, campesinos y políticas agrarias en Colombia 1920-1980, Bogotá, Universidad Nacional de Colombia-CID.

Fals-Borda, Orlando (1982), Historia de la cuestión agraria en Colombia, Bogotá, Carlos Valencia Editores.

Forero, Édgar (2003), El desplazamiento interno forzado en Colombia, en <www.ideaspaz.org/publicaciones>, 20 de marzo.

Fukuyama, Francis (1992), El fin de la historia y el último hombre, México, Planeta.

Fundación Universitaria Luis Amigó-Sede de Montería (2003), "Diagnóstico socioeconómico sobre el mototaxismo", manuscrito no publicado.

Gaviria, Alejandro (2002), Ley 789 de 2002, funcionó o no, en www.banrep.gov.com, 4 de marzo.

Gutiérrez-Castro, Édgar (2002), "Relato de un difícil rescate", en Alberto Baquero (coord.), Modelos de desarrollo económico en Colombia, Bogotá, Oveja Negra.

Helmsing, A. H. J. Bert (1999), “Teorías de desarrollo industrial regional y políticas de segunda y tercera generación”, Revista EURE XXV(75), Santiago de Chile, pp. 5-39. 
Hernández-Pérez, Verena (2004), "Un enfoque marxista de la globalización”, ponencia presentada en II Congreso Internacional sobre las obras de Carlos Marx, Ministerio de Educación Superior, La Habana, en www.rebelion.org.

Hommes, Rudolf (2002), "La apertura y las reformas económicas”, en Alberto Baquero (coord.), Modelos de desarrollo económico en Colombia 1960-2002, Bogotá, Oveja Negra.

López G., Luis Fernando (1995), Intervencionismo de Estado y economía en Colombia, Bogotá, Universidad Externado de Colombia.

Kalmanovitz, Salomón, (1978), El desarrollo capitalista en el campo colombiano, Bogotá, Colombia Hoy.

Kalmanovitz, Salomón (2001), Las instituciones y el desarrollo económico en Colombia, Bogotá, Norma.

Ley 101 de 1993, Diario Oficial, núm. 41,149 del 23 de diciembre, Bogotá.

Ley 160 de 1994, Diario Oficial, núm. 41,479 del 3 de agosto, Bogotá.

Machado, Absalón (s.f.), "Tenencia de la tierra, problema agrario y conflicto", en http://www.virtual.unal.edu.co/cursos/ humanas/2004945/docs_Absalon\%20Machado.pdf.

Mattos, Carlos de (2000), "Nuevas teorías del crecimiento económico: una lectura desde la perspectiva de los territorios", Revista de Estudios Regionales, 58, Universidad Complutense de Madrid en <http://www.ucm.es/BUCM/ compludo >, pp.15-36.

Ministerio de Comercio Exterior de Colombia (2001), Perfil Departamental Córdoba, Bogotá, Ministerio de Comercio Exterior de Colombia.

Misión para el Diseño de Estrategias de la Red de Pobreza y la Desigualdad (2004) en www.asocajas.org.co/2005/Documentos/Otros/MISIÓN\%20POBREZA 
Moncayo, J. Édgar (2002), “Nuevos enfoques de política regional en América Latina: el caso de Colombia en perspectiva histórica”, Las políticas regionales en Colombia, separata 5, Documento 198, DNP, Bogotá, Archivos de Economía.

Ocampo, José Antonio (1992), "Reforma del Estado y Desarrollo Económico y Social en Colombia" Revista análisis político,17, Instituto de Estudios Políticos y Relaciones Internacionales Universidad Nacional de Colombia, Bogotá, Colombia, pp 5-40.

PNUD-DNP (2003), Diez años de desarrollo humano en Colombia, Bogotá, PNUD-DNP.

Ramos-Hernández, Maribel (s.f.), "Pobreza: definiciones internacionales y alternativas metodológicas", <www.nodo 50org/Cubasigloxxi>, 11 de noviembre.

Rodríguez, Karen (2004), "Medición del impacto de un programa de reforma agraria en Colombia", en <www.ideas.repec. org/s/col/000089.html>

Romero-Reyes, Antonio (2003), "Economía política de la globalización capitalista", en <www.rebelion.org $>, 2$ de octubre.

Romero Gómez, Antonio (2000), “Globalización y economía internacional. Un análisis desde la perspectiva del desarrollo", ponencia presentada en el Cuarto Encuentro Iberoamericano de Estudios Prospectivos, La Habana.

Sánchez-Juliao, David (1975), Historias de Raca Mandaca, Barranquilla, Publicaciones de la Rosca.

Sánchez, Ricardo (1984), Estado y planeación en Colombia, Medellín, Bedout.

Sarmiento, Libardo (2005), "Colombia: crecimiento sin bienestar”, en www.rebelion.org, febrero 2005.

Solow, Robert M (1956), "A Contribution to the Theory of Economic Growth" The QuarterlyJournal of Economics, Vol. 
70, 1, Feb. pp 65-94. Traducción al Castellano en Sen (1979) como "Un Modelo de Crecimiento", pp. 151-182.

Swan, Trevor W(1956), "Economic Growth and Capital Accumulation" Economic Record, 32, pp. 334-361, Corporación Autónoma Regional del Valle del Cauca, 1954.

Triveli-O., Pablo (1995), "Documento técnico sobre la ciudad latinoamericana: gestión urbana para el siglo XXI, retos y propuestas", ponencia presentada en el Encuentro Latinoamericano y del Caribe de Alcaldes preparatorio de Hábitat II, Río de Janeiro, Brasil, 10 de junio de 1995.

Recibido: 6 de octubre de 2006. Aceptado: 30 de abril de 2007.

Jorge Eliécer Ortega Montes. Es magíster en planeación urbana por la Universidad Nacional de Colombia, Sección Medellín. Ha sido becario por la Corporación Autónoma Regional de los Valles del Sinú y de San Jorge. Posgraduado en planificación del desarrollo rural regional integrado del Centro de Estudios Regionales Urbano Rurales (CERUR), Rehoboth, Israel, becado por la Embajada del Estado de Israel. Actualmente es director del grupo de investigación Desarrollo, Democracia y Planeación Local Regional, reconocido por Colciencias, con las siguientes líneas de investigación: educación y desarrollo local, gestión y estudios urbanos y regionales, pobreza, desigualdad y desarrollo humano. Entre sus publicaciones destacan Alternativa agroindustrial para el aprovechamiento de los excedentes de la producción de plátano en el Departamento de Córdoba, Bogotá, Editora Guadalupe, 94 pp. (2004); Plan de desarrollo regional provincias de Sara e Ichilo, departamento de Santa Cruz, Bolivia, Rehovoy, Israel, Centro de Estudios Regionales, 254 pp. (1993); Propuesta de un modelo de planeación participativa para las regiones del Sinú y San Jorge, departamento de Córdoba, Bogotá, Centro de Publicaciones de la Escuela Superior de Administración Pública (ESAP), 205 pp. (1992). 